\title{
Low recurrence rate of a two-layered closure repair for primary and recurrent midline incisional hernia without mesh
}

\author{
A. H. M. Dur · D. den Hartog • W. E. Tuinebreijer • \\ R. W. Kreis $\cdot$ J. F. Lange
}

Received: 4 November 2008 / Accepted: 13 February 2009 / Published online: 19 March 2009

(C) The Author(s) 2009. This article is published with open access at Springerlink.com

\begin{abstract}
Background Incisional hernia is a serious complication after abdominal surgery and occurs in 11-23\% of laparotomies. Repair can be done, for instance, with a direct suture technique, but recurrence rates are high. Recent literature advises the use of mesh repair. In contrast to this development, we studied the use of a direct suture repair in a separate layer technique. The objective of this retrospective observational study is to assess the outcomes (recurrences and complications) of a two-layered open closure repair for primary and recurrent midline incisional hernia without the use of mesh.

Methods In an observational retrospective cohort study, we analysed the hospital and outpatient records of 77 consecutive patients who underwent surgery for a primary or recurrent incisional hernia between 1st May 2002 and 8th November 2006. The repair consisted of separate continuous suturing of the anterior and posterior fascia, including the rectus muscle, after extensive intra-abdominal adhesiolysis.

Results Forty-one men (53.2\%) and 36 women (46.8\%) underwent surgery. Sixty-three operations $(81.8 \%)$ were primary repairs and 14 (18.2\%) were repairs for a recurrent incisional hernia. Of the 66 patients, on physical examination, three had a recurrence (4.5\%) after an average follow-up of
\end{abstract}

D. den Hartog and A.H.M. Dur contributed equally to this article.

A. H. M. Dur · W. E. Tuinebreijer · R. W. Kreis

Department of Surgery, Red Cross Hospital,

Vondellaan 13, 1942 LE Beverwijk, The Netherlands

D. den Hartog $(\varangle)$ · J. F. Lange

Department of Surgery, Erasmus Medical Center,

University Medical Center Rotterdam, 's-Gravendijkwal 230,

Office H-960, 3000 CA Rotterdam, The Netherlands

e-mail: d.denhartog@erasmusmc.nl
2.6 years. The 30 -day postoperative mortality was $1.1 \%$. Wound infection was seen in five patients $(6.5 \%)$.

Conclusions A two-layered suture repair for primary and recurrent incisional hernia repair without mesh with extensive adhesiolysis was associated with a recurrence rate comparable to mesh repair and had an acceptable complication rate.

Keywords Ventral hernia - Abdominal hernia . Suture repair $\cdot$ Direct repair $\cdot$ Recurrence

\section{Introduction}

Incisional hernias are ventral hernias through an operation scar and are a serious complication of abdominal surgery. Incisional hernias occur in $11-23 \%$ of laparotomies [1]. Incisional hernias enlarge over time and can give rise to such complications as pain, discomfort, bowel obstruction, incarceration and strangulation. Furthermore, incisional hernias reduce the quality of life and the chances for employment.

The repair can be done by either an open or a laparoscopic technique. The open technique can be a simple hernioplasty (Mayo duplication or fascia-adaptation), a components separation technique or a mesh repair. Laparoscopic correction is always performed with a mesh. The recurrence rate after open suture repair may be as high as $54 \%$ [2], and for open mesh repair, up to $32 \%$ [2, 3]. The recurrence rate for laparoscopic repair appears to be comparable to the open mesh procedure, but with a shorter hospital stay [1]. In a Swedish cost analysis study (including sick leave), the costs for incisional hernia repair by suture were 6122 Euro and for repair with mesh, they were 5458 Euro [4]. 
In a recent Cochrane review, the authors conclude that mesh repair is superior to suture repair in terms of recurrences [5]. Burger et al. [6] stated that the suture repair of incisional hernias should be completely abandoned. However, in our experience, dedicated surgery for incisional hernias justifies the direct suture repair in a separate layer technique without the use of mesh. In contrast to the mesh repair, the infection rate after suture repair is lower [6]. Furthermore, the clinical relevance of (partial) radiological recurrences is uncertain [5] because, in the literature, imaging techniques are only used in case of doubt.

The objective of this retrospective observational study is to assess recurrences and complications of our two-layered closure repair for primary and recurrent incisional hernia and to delineate the relationship between clinical and radiological recurrences.

\section{Patients and methods}

We conducted a retrospective study of incisional hernias repaired with a two-layered closure method at the Red Cross Hospital, Beverwijk, The Netherlands, between 1st May 2002 and 8th November 2006. The hospital information system was used to find all patients who had undergone an operation for primary or recurrent incisional hernia. We obtained all of the information from the hospital and outpatient medical records. We identified 77 consecutive cases. Six patients were deceased, of which one death was related to the operation (30-day mortality). In January 2008, 71 patients were asked to attend a physical and an ultrasound examination. Fifteen patients were unable to attend the ultrasound examination, despite repeated attempts. Four patients reached the endpoint of the study because of a reoperation for a reason other than recurrence. These patients were included in the physical examination group for calculating the recurrence rate.

A two-layered closure repair was performed without the use of mesh in 77 patients: 36 women $(46.8 \%)$ and 41 men $(53.2 \%)$. The mean age of these patients was 62.2 years (standard deviation $[\mathrm{SD}]=14.4$ ). The mean body mass index (BMI) was $28.8 \mathrm{~kg} / \mathrm{m}^{2}(\mathrm{SD}=6.5)$. The indication was a primary incisional hernia in 63 patients $(81.8 \%)$ and a recurrent incisional hernia in 14 patients $(18.2 \%)$. The primary hernia repair in the 14 patients with a recurrent repair was a suture repair in nine patients and a mesh repair in five patients. A history of smoking was found in 19 patients $(24.7 \%)$, diabetes in nine patients $(11.7 \%)$ and corticosteroid use in two patients $(2.6 \%)$. A wound infection was found in 15 patients $(19.5 \%)$ after the primary operation after which the incisional hernia developed. In nine patients, a history of a burst abdomen was found (11.7\%).
The hernias were measured during operation and classified according to size in three categories: small $(<5 \mathrm{~cm}$ in width or length), medium (5-10 cm in width or length) and large ( $>10 \mathrm{~cm}$ in width or length) [7].

All US examinations were done by one radiologist. Ultrasound examinations were performed using high-end ultrasound equipment (Aplio XG, model SSA-796A, Toshiba Medical Systems Corporation, 1385, Shimoishigami, Otawara-Shi, Tochigi-Ken 324-8550, Japan, and ATL 5000, Philips, ATL Factories, Bothell, WA, USA). A total recurrence was defined as a defect in both the posterior and the anterior fascia.

Operations were performed either by one of two surgeons or by surgical residents under the supervision of these two surgeons.

\section{Operative technique}

The abdomen is opened through the midline incision scar. The incision is equal to the length of the scar and does not depend on the size of the hernia. All scar tissue is excised. The mutual bowel adhesions and adhesions between the bowel and the ventral abdominal wall are removed. In this manner, there is no retraction of the abdominal wall and the bowels are divided equally over the whole abdominal cavity. The hernia sac and scar tissue are excised from the fascia rims. On both sides of the incision, the rectus sheath is opened from the midline to develop a free anterior and posterior fascia of the same length. The rectus muscles become exposed during this manoeuvre. With a running polydioxanone suture (PDS loop, Ethicon, Johnson \& Johnson Medical), the posterior fascia is closed together with the peritoneum, and parts of the rectus muscle are included. The suture technique is done with a short stitch length and a suture-length-to-wound-length ratio of four or more, as described by Millbourn and Israelsson [8]. Next, the anterior fascia is closed with a running polydioxanone suture, and parts of the rectus muscle are included. The rectus muscles are not sutured together separately, but always with the anterior or posterior fascia. At the end of this procedure, two separate layers are identified: the anterior and posterior fascia with the rectus muscles. Mesh and drains are not used as a standard procedure. If the tension on the fascia is too tight, the anterior and posterior fasciae are closed simultaneously to reduce the tension on the posterior fascia. Additionally, the closure is simultaneously started superiorly and inferiorly to divide the tension over the entire wound. In most of our cases, it is not a tension-free technique. Subcutaneous tissue and skin are closed with interrupted sutures. Surplus skin arising as a consequence of this anatomical repair is excised. The surgical technique is shown in Figs. 1-4. 


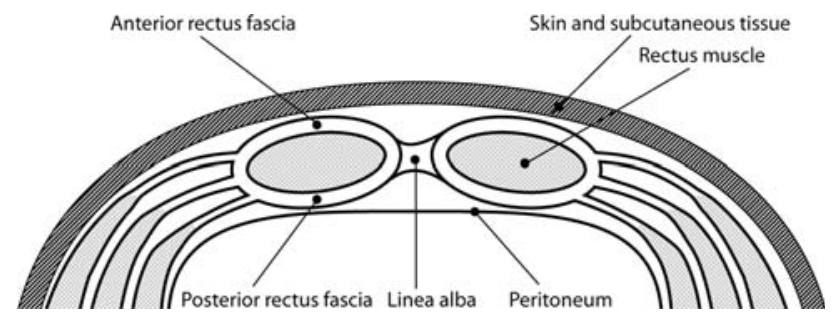

Fig. 1 Diagram of the anatomy of the median ventral abdominal wall. In front of the rectus muscles are the anterior rectus fasciae and on the backside the posterior rectus fasciae. The anterior and posterior rectus fasciae join medially to form the linea alba

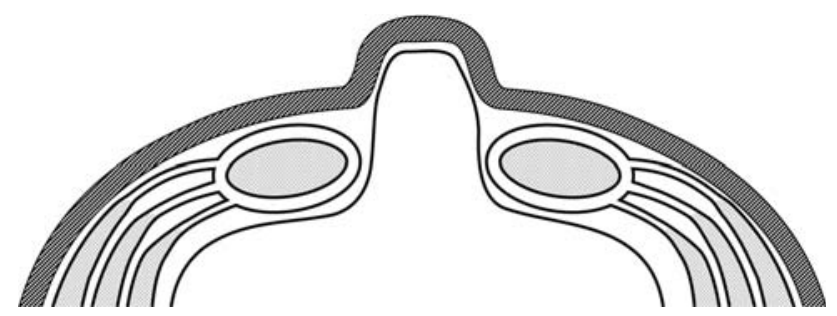

Fig. 2 Diagram of the anatomy of an incisional hernia through a midline abdominal incision. The hernial sac consists of the peritoneum, which is covered by skin and subcutaneous tissue

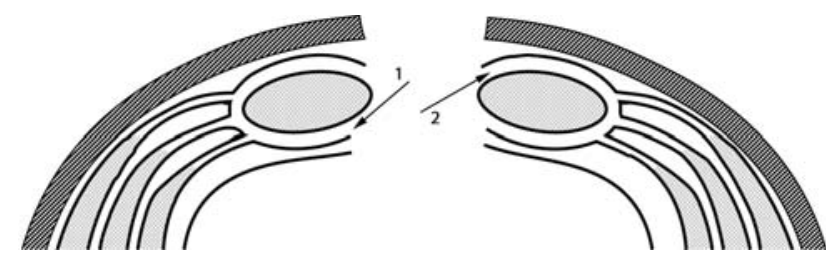

Fig. 3 Diagram of the surgical situation after resection of the scar tissue and hernial sac. The rectus sheaths are opened through the posterior rectus fasciae (arrow 1) on the medial sides. Then, the rectus muscles and anterior rectus fasciae (arrow 2) are exposed

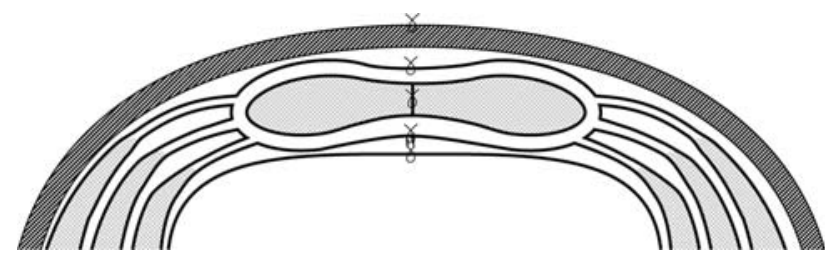

Fig. 4 Diagram of the three-layered closure. Anterior and posterior rectus fasciae and rectus muscles are sutured in separate layers and form the three layers of the repair

\section{Statistical analysis}

The statistical analysis was performed with SPSS (version 11.0) on a personal computer. Categorical data are presented as the number of subjects in the category, along with the percentages. All continuous data are given as means with SDs.

The Chi-square test and Fischer's exact test were used to compare the patients with and without an ultrasound follow-up examination and the patients with and without a recurrence. The independent Student's $t$-test was used to compare the length of follow-up of the patients with and without an ultrasound follow-up examination. A $P$-value $<0.05$ will be taken as the threshold of statistical significance.

\section{Results}

The mean operation time was $114.5 \mathrm{~min}(\mathrm{SD}=65.7)$. The mean blood loss was $438.2 \mathrm{ml}(\mathrm{SD}=842.7)$. The mean postoperative stay in the hospital was 9.0 days $(\mathrm{SD}=13.4)$ and the mean stay in the intensive care unit (ICU) was 1.1 days $(\mathrm{SD}=4.4)$. The mean ventilation time in days in the ICU was 0.68 days $(\mathrm{SD}=2.9)$. Seven patients $(9.1 \%)$ were ventilated and 14 patients $(18.2 \%)$ were admitted to the ICU. Admission to the ICU was often preoperatively planned by the anaesthesiologist because of preoperatively estimated comorbidity. The mean length of follow-up for the ultrasound examination was 3.1 years $(\mathrm{SD}=0.94$, range 1.2-5.2 years).

The primary operation after which the incisional hernia developed was a median upper abdominal incision in 18 patients (23.4\%), a median lower abdominal incision in 13 patients (16.9\%) and a combined upper and lower incision $(59.7 \%)$ in 46 patients.

The size of the hernia was small in 20 patients $(26.7 \%)$, medium in 41 patients $(54.7 \%)$ and large in 14 patients $(18.7 \%)$. Information about the size of the hernia was missing in two patients.

The complications are stated in Table 1. The 30-day postoperative mortality was $1.3 \%(n=1)$. The cause of

Table 1 Complications

\begin{tabular}{ll}
\hline Mortality & $1(1.3 \%)$ \\
Wound infection & $5(6.5 \%)$ \\
Wound haematoma & $2(2.6 \%)$ \\
Respiratory insufficiency & $4(5.2 \%)$ \\
Pneumonia & $5(6.5 \%)$ \\
Cardiovascular complications & $6(7.8 \%)$ \\
Bowel obstruction & $2(2.6 \%)$ \\
Wound fistula & $1(1.3 \%)$ \\
Abdominal compartment syndrome & $1(1.3 \%)$ \\
Recurrence & \\
By physical examination & \\
Yes & $3(4.5 \%)$ \\
No & $63(95.5 \%)$ \\
By ultrasound examination & \\
Yes & $7(13.5 \%)$ \\
No & $44(84.6 \%)$ \\
\hline
\end{tabular}


mortality was aspiration followed by pneumonia and multiple organ failure. Wound infection was seen in five patients $(6.5 \%)$ and wound haematoma was seen in two patients (2.6\%). Respiratory insufficiency was seen in four patients $(5.2 \%)$ and pneumonia was seen in five patients $(6.5 \%)$, leading to artificial ventilation in three patients, with full recovery. Cardiovascular complications were seen in six patients $(7.8 \%)$ and were treated with medication and follow-up by the cardiologist. Three patients experienced cardiovascular complications with respiratory insufficiency, including pneumonia. Temporary bowel obstructions were seen in two patients $(2.6 \%)$ and were treated conservatively. Wound fistula developed in one patient (1.3\%). The wound healed without further complications after exploration with the removal of a suture granuloma. An abdominal compartment syndrome was seen in one patient (1.3\%), which necessitated immediate open-abdomen treatment and successive closure with sublay mesh technique.

Of the 77 patients, six deceased, one was unable to attend follow-up physical examinations and 15 were unable to attend the ultrasound examination, despite repeated attempts. Four patients reached the endpoint of the study because of re-operation for a reason other than recurrence. These four patients were included in the physical examination group for calculating the recurrence rate. In this group, one recurrence was diagnosed during surgery.

No significant differences were found between the patients with and without an ultrasound follow-up examination for the variables examined, except for a significant difference for sex and length of follow-up of the physical examination. More women than men had an ultrasound follow-up examination $(P=0.002)$. The mean length of follow-ups for the physical examination in the ultrasound and non-ultrasound groups were 3.1 years $(\mathrm{SD}=0.94)$ and 1.15 years $(\mathrm{SD}=1.23)$, respectively $(P=0.000)$. Recurrences were diagnosed by physical examination in three patients out of 66 patients examined $(4.5 \%)$ after a mean follow-up of 2.6 years $(\mathrm{SD}=1.3)$, and by ultrasound in seven patients out of 52 patients examined (13.5\%) after a mean follow-up of 3.1 years $(\mathrm{SD}=0.94)$. All of these patients had a partial defect in the length of the fascia. One patient had only a small defect of the posterior fascia of the rectus sheath with an intact anterior fascia diagnosed by ultrasound. There were no symptoms and no recurrences diagnosed by physical examination in this patient, and the data of this patient were not used for the calculation of the recurrence rate after ultrasound, given our definition of recurrence.

Predictor variables of hernia recurrences were not found and probably could not be estimated because of the low recurrence and complication rates. For instance, no correlations were found between recurrence and hernia size, BMI, age, sex, diabetes and smoking.

\section{Discussion}

Our two-layered suture repair of incisional hernias without mesh showed a clinical recurrence rate of $4.5 \%$ after a mean follow-up of 3.1 years. We found a discrepancy between the clinical and ultrasound recurrence rates. Of 52 patients, examination with ultrasound identified seven with a recurrence $(13.5 \%)$ after a mean follow-up time of 3.1 years. Most publications only report the lower clinical recurrence rates, which will be more strongly correlated with the complaints and symptoms of the patient. In four recent randomised clinical trials (RCTs) investigating incisional hernias, the outcome of recurrence was measured by physical examination, and radiological examination was done only on indication, but the recurrence rates by these examinations were not reported [6, 9-11].

Our complication rate was acceptable. For instance, our wound infection rate was $6.5 \%$. A recent Cochrane review calculated a pooled infection rate for mesh repair of $10.1 \%$ [5]. In mesh repair, wound infection can lead to infection of the prosthesis, sometimes necessitating mesh removal.

The Cochrane review found solid evidence to advocate for the use of mesh repair for the open repair of incisional hernias [5]. The pooled recurrence rate was $33.3 \%$ for the suture repair group and $16.4 \%$ for the mesh group. In the $\mathrm{RCT}$ of Burger et al., the recurrence rate in the suture group was $56 \%$ after a median follow-up of 75 months, and in the RCT of Korenkov et al., the recurrence rate was $12 \%$ after 13 months [6, 11].

On a poster, Baracs et al. [12] reported the results of a multicentre randomised trial. The recurrence rate was $16.9 \%$ in the suture group $(n=89)$ compared with $6.8 \%$ in the sublay mesh group $(n=103)$ after a follow-up of $3-$ 5 years; this difference was significant.

In their study of giant hernias with a length of at least $20 \mathrm{~cm}$, de Vries Reilingh et al. [9] described a high recurrence rate of $56 \%$ in the components separation group. The authors could not close the fascia in their patients with a mesh repair. With the two-layered closure technique, it was possible to close the fascia in our patients. Our study group included different hernia sizes, but most hernias (54.7\%) were between 5 and $10 \mathrm{~cm}$ in width or length and $18.7 \%$ were larger than $10 \mathrm{~cm}$ in width or length. This distribution was caused by the fact that our patients are a continuous series and that our hospital is a referral centre for incisional hernias. This diverse distribution of the hernia sizes and our inclusion of patients consecutively in this study allowed us to conclude that there was no patient selection.

What makes our technique so successful? The following reasons might explain our success. First, the two-layered suture repair consists of an extensive adhesiolysis, which prevents retraction and gives space to move the abdominal wall to the midline. In addition, the adhesiolysis makes it 
possible for the bowels to move freely instead of moving as a block, and they adjust more easily to the reduction in the abdominal cavity. Second, the abdominal wall is anatomically reconstructed. It is very important that the rectus muscles are placed in their normal median position. In our technique, they are attached to each other at the midline and, as a result, they can exert normal function. It is important to note that we do not suture the rectus muscles separately to each other, but always together with the anterior or posterior fascia to prevent tension and, thus, necrosis of the muscle.

Muscle cannot stretch as fascia does, and muscle can keep an isometric state during different loads, such as lifting. Furthermore, the two-layered fascia closure is stronger than a one-layered repair. Another explanation relies on theoretical arguments that fibroblasts from the rectus muscle positively influence the fascia healing in the midline. All scar tissue and the hernia sac are additionally removed up to the median border of the rectus fascia. Finally, the entire primary incision is explored independently of the size of the hernia, so we are treating future recurrences along these parts of the fascia. Often, weak spots and small defects are found in the fascia that are distinct from the repaired hernia. As a result, we only use healthy functional tissue and simultaneously identify non-diagnosed small hernias within the remainder of the scar. Because the anterior and posterior fascia are often sutured under tension, we abandon the important surgical principle in hernia surgery of tension-free repair. Our low recurrence rate shows that this is possible. However, the disadvantage of this technique under tension can be the occurrence of an abdominal compartment syndrome, which, in our series, was treated by open abdomen and successively closed with the mesh technique.

An important observation to explain our low recurrence rate is the low incidence rate of incisional hernias $(<1 \%)$ in the so called lateral paramedian incision [13]. In this incision, the anterior and posterior rectus sheath is incised at a point not less than two-thirds of the width of the rectus sheath from the midline. The sutured rectus sheaths are covered by rectus muscle and, therefore, are comparable with our two-layered suture repair.

All of our surgeries were done by or under the direct supervision of experienced surgeons, using a strict protocol for the surgical technique. Our good results could be due, in part, to this method of dedicated surgery. In contrast, in all of the referenced RCTs, the open suture repairs are inappropriately described in this regard $[6,11,12]$.

This operation is a major procedure that can result in a longer hospital stay, intensive care stay and time on a ventilator.

What is significant is that more women than men attended the ultrasound examination, which we explained as a better compliance and cooperation of the female patients. The longer length of follow-up for the physical examination of the patients with an ultrasound examination can be explained by the fact that the ultrasound examination was always preceded by a physical examination by the surgeon. So the willingness of the patient to come to the hospital for the ultrasound examination was used to perform a physical examination. The ultrasound examination was performed at the end of this study and can be viewed upon as the prospective part of our study. However, these two significant findings could also be false-positive results of the subgroup analysis.

Because our study is retrospective, it could be open to bias. For this reason, it is necessary to perform an RCT comparing our technique with mesh repair. In contrast to our technique, the latter can be done laparoscopically with less blood loss and a shorter hospital stay [14, 15]. An important advantage of the direct suture repair is the avoidance of using a mesh. This avoids the risk of prolonged pain, seroma and mesh-related infections, which often require mesh removal [14]. In one study, extensive laparoscopic adhesiolysis was the major cause of an enterotomy rate of $3.3 \%$, which often necessitates conversion to an open procedure [14]. The complications of the mesh repair should be weighed against the $1.3 \%$ risk of occurrence of an abdominal compartment syndrome in our study. By avoiding the use of a mesh prosthesis, which will often be represented by an expensive intraperitoneally positioned composite mesh, a very important cost reduction can be accomplished. In contrast with our anatomical repair, the laparoscopic repair has, as a common problem, the surplus of skin that remains and chronic postoperative seroma.

We advocate the use of the two-layered closure technique for primary and recurrent incisional hernias without a mesh.

Open Access This article is distributed under the terms of the Creative Commons Attribution Noncommercial License which permits any noncommercial use, distribution, and reproduction in any medium, provided the original author(s) and source are credited.

\section{References}

1. Cassar K, Munro A (2002) Surgical treatment of incisional hernia. Br J Surg 89(5):534-545

2. Paul A, Korenkov M, Peters S, Köhler L, Fischer S, Troidl H (1998) Unacceptable results of the Mayo procedure for repair of abdominal incisional hernias. Eur J Surg 164(5):361-367

3. Burger JW, Lange JF, Halm JA, Kleinrensink GJ, Jeekel H (2005) Incisional hernia: early complication of abdominal surgery. World J Surg 29(12):1608-1613

4. Israelsson LA, Jönsson L, Wimo A (2003) Cost analysis of incisional hernia repair by suture or mesh. Hernia 7(3):114-117

5. den Hartog D, Dur AHM, Tuinebreijer WE, Kreis RW (2008) Open surgical procedures for incisional hernias. Cochrane Database Syst Rev 3:CD006438 
6. Burger JW, Luijendijk RW, Hop WC, Halm JA, Verdaasdonk EG, Jeekel J (2004) Long-term follow-up of a randomized controlled trial of suture versus mesh repair of incisional hernia. Ann Surg 240(4):578-583

7. Korenkov M, Paul A, Sauerland S, Neugebauer E, Arndt M, Chevrel JP, Corcione F, Fingerhut A, Flament JB, Kux M, Matzinger A, Myrvold HE, Rath AM, Simmermacher RK (2001) Classification and surgical treatment of incisional hernia. Results of an experts' meeting. Langenbecks Arch Surg 386(1):65-73

8. Millbourn D, Israelsson LA (2004) Wound complications and stitch length. Hernia 8(1):39-41

9. de Vries Reilingh TS, van Goor H, Charbon JA, Rosman C, Hesselink EJ, van der Wilt GJ, Bleichrodt RP (2007) Repair of giant midline abdominal wall hernias: "components separation technique" versus prosthetic repair: interim analysis of a randomized controlled trial. World J Surg 31(4):756-763

10. Conze J, Kingsnorth AN, Flament JB, Simmermacher R, Arlt G, Langer C, Schippers E, Hartley M, Schumpelick V (2005) Randomized clinical trial comparing lightweight composite mesh with polyester or polypropylene mesh for incisional hernia repair. Br J Surg 92(12): 1488-1493

11. Korenkov M, Sauerland S, Arndt M, Bograd L, Neugebauer EA, Troidl H (2002) Randomized clinical trial of suture repair, polypropylene mesh or autodermal hernioplasty for incisional hernia. Br J Surg 89(1):50-56

12. Baracs J, Weber G, Takacs I, Horvath OP (2007) Results of open mesh versus suture mesh repair in treatment of abdominal wall hernias. In: Proceedings of the 29th International Congress of the European Hernia Society, Athens, Greece, May 2007

13. O'Dwyer PJ, Courtney CA (2003) Factors involved in abdominal wall closure and subsequent incisional hernia. Surgeon 1(1):17-22

14. Perrone JM, Soper NJ, Eagon JC, Klingensmith ME, Aft RL, Frisella MM, Brunt LM (2005) Perioperative outcomes and complications of laparoscopic ventral hernia repair. Surgery 138(4):708-715

15. Pierce RA, Spitler JA, Frisella MM, Matthews BD, Brunt LM (2007) Pooled data analysis of laparoscopic vs. open ventral hernia repair: 14 years of patient data accrual. Surg Endosc 21(3):378386 